\title{
Chapter 23 \\ Citizen Science in the Digital World of Apps
}

\author{
Rob Lemmens, Vyron Antoniou, Philipp Hummer, and Chryssy Potsiou
}

\begin{abstract}
In this chapter, we highlight the added value of mobile and web apps to the field of citizen science. We provide an overview of app types and their functionalities to facilitate appropriate app selection for citizen science projects. We identify different app types according to methodology, data specifics, and data collection format.

The chapter outlines good practices for creating apps. Citizen science apps need to ensure high levels of performance and usability. Social features for citizen science projects with a focus on mobile apps are helpful for user motivation and immersion and, also, can improve data quality via community feedback. The design, look and feel, and project identity are essential features of citizen science apps.

We provide recommendations aimed at establishing good practice in citizen science app development. We also highlight future developments in technology and, in particular, how artificial intelligence (AI) and machine learning (ML) can impact citizen science projects.
\end{abstract}

Keywords Mobile apps · Software development · Data collection

\footnotetext{
R. Lemmens $(\triangle)$

Faculty of Geo-Information Science and Earth Observation (ITC), University of Twente,

Enschede, The Netherlands

e-mail: r.l.g.lemmens@utwente.nl

V. Antoniou

Hellenic Army Geographic Directorate, Athens, Greece

P. Hummer

SPOTTERON Citizen Science, Vienna, Austria

C. Potsiou

School of Rural \& Surveying Engineering, National Technical University of Athens, Athens, Greece
} 


\section{Introduction}

The widespread use of smartphones has created new opportunities in the field of citizen science (Silvertown 2009; Newman et al. 2012; Bonney et al. 2014; Wynn 2017). Mobile apps provide a new way to steer the data gathering process as part of the scientific method. Communication with and among participants is now possible at any time, as many people carry their smartphones with them almost constantly. In addition, smartphone sensors offer new possibilities for comprehensive and comparable data collection (Teacher et al. 2013).

The term app refers to a wide range of software, running on mobile devices, in browsers, on desktops, and even on smartwatches. In citizen science, applications are most commonly referred to as apps, either for smartphones (mobile apps) or for websites, accessed via desktops or laptops (web apps). These apps can form part of the support infrastructure in citizen science, so-called citizen science platforms (see Hai-Ying Liu et al., this volume, Chap. 22), which provides tools and facilitates communication and interaction between participants.

Apps dramatically extend the feature set of a classic website and increase interactivity and participation options. The smartphone has brought about a revolution in citizen science. With mobile devices, citizens now have constant access to what are effectively microcomputers equipped with sensors; these are connected to the web and are always ready for use. Mobile apps have enabled direct participation. They can also help to overcome the obstacles of location and time in certain citizen science tasks (Sturm et al. 2017). As smartphones and their user interfaces have become mainstream technology, people are able to interact more intuitively with their installed apps.

\section{The Use of Apps in Citizen Science}

\section{Why Apps?}

The revolutionary uptake of the smartphone has changed our daily lives in many respects. Mobile technology facilitates participation in interactive platforms and projects. For citizen science, the deployment of mobile apps means that participants can contribute observations in real time. To report relevant sightings immediately not only increases the quality of the provided data (in terms of timeliness) but also improves the connection of the observer with the subject and its environment, as the observer is immersed in the field and is aware of the context of their observations.

Furthermore, mobile apps are important digital tools that integrate data coming from sources in real time. They can enrich information provision, not only for the younger generations but also for the majority of the public. Especially for digital natives (the generation who grew up with digital technology), a mobile app offers the means of participation via using the interactive features of their smartphones. 


\section{More than Data Collection}

According to the ECSA (2015), citizen science involves more than just data collection. While the data set is one of the main outcomes when running a citizen science (software) toolkit, this is often not the core focus. Citizen science is based on participatory principles, which not only position the public in a data collection role but also encourage volunteers to join in the quest of solving scientific challenges (Haklay 2013). Taking part in the scientific process stimulates open data access and reproducible and collaborative research, raises public awareness, and generally empowers citizens (Trojan et al. 2019). The level of participation in the scientific process depends on the citizen science approach utilised (e.g. raw data collection versus interpreting observations). In digital approaches, such as app toolkits, this diversifies the roles available for volunteers. Achieving this necessitates providing a wide range of interactive features within an app, for example, facilitating data entry via menus, free text, sensor interfaces, etc. The decision to use a mobile or deskbased data collection method depends on (1) if there is a need to capture field observations; (2) whether the application needs to be frequently accessible to the participant; and (3) how much data analysis is involved. In the first two cases, a mobile app is preferable; and in the last case, a web app is preferable.

As interactive digital apps with user-generated content (UGC), citizen science apps have strong links to social media approaches. Migrating social media elements to citizen science apps can significantly increase their effectiveness. For example, citizen science apps can mimic social media by allowing volunteers to create groups and subgroups depending on their interests. This can help to promote the aims of citizen science projects, such as allowing direct communication among users in order to clarify uncertainty about project processes, micro-management of niche processes, and sharing achievements with broader audiences to raise awareness (Ambrose-Oji et al. 2014; Luna et al. 2018).

Citizen science apps can also include external data, either from other digital sources or from attached sensors. With this approach, apps are used as visualisation interfaces for monitoring or viewing data, rather than for active data collection.

Like all apps, citizen science apps are subject to user expectations (Pejovic and Skarlatidou 2020) and should therefore incorporate the following core requirements:

- Usability: the ease of use for the participants

- Look and feel: the visual quality of an app

- Performance: the speed with which an app opens and operates

- Security: the level of technical security and encryption of sensitive data

- Compatibility: the range of operating systems and devices supported

- User privacy: compliance with the EU General Data Protection Regulation (GDPR)

Gamification can also be a valid approach for certain citizen science projects. Participant motivation can be fostered by applying game-like elements and inviting competition between users (Bowser et al. 2013). Examples are bio-puzzles like 
Foldit, EyeWire, Stall Catchers, and others available on the Citizen Science Games platform.

\section{Project Examples}

Mobile apps are developed for many purposes. Some of the most common functionalities of mobile app-based platforms include:

- Surveys: often website based, surveys ask users a range of hierarchical questions about a specific topic.

- Spotting: map-based contributions of topic-related observations, most commonly logged by participants on smartphones.

- Sensing: sensor observations obtained with sensors internal or external to the mobile phone.

- Image and video classification: classification activities in an array of images or videos.

- Gaming: citizen science games for data generation, often with a competitive aspect.

In Table 23.1 we highlight some prominent examples of projects in which mobile apps play diverse roles.

Table 23.1 Examples of mobile apps in citizen science projects

\begin{tabular}{|c|c|}
\hline App & Functionality \\
\hline $\begin{array}{l}\text { Mobile app: SpiderSpotter } \\
\text { In the SpiderSpotter citizen science app, powered by SPOTTERON, users } \\
\text { contribute observations of spiders and their webs to help research their adaptation } \\
\text { to the environment. The app was launched with a highly successful campaign } \\
\text { involving newspapers and radio stations in Belgium with thousands of app } \\
\text { downloads in the first few days followed by many contributions from the public. } \\
\text { The complete citizen science application consists of mobile apps, a web app for } \\
\text { browsers, a data administration interface, and a special toolkit for data analysis } \\
\text { and visualisation. This special toolkit allows users to record size and colour } \\
\text { measurements of data collected by citizen scientists and to interactively visualise } \\
\text { the analysed data on maps }\end{array}$ & Spotting \\
\hline $\begin{array}{l}\text { Citizen science game: Stall Catchers } \\
\text { Stall Catchers is an online game that anyone can play without any prior experi- } \\
\text { ence. In the game, participants look at movies from the brains of mice and try to } \\
\text { identify clogged blood vessels or stalls. The aim is to help facilitate Alzheimer's } \\
\text { disease research at Cornell University }\end{array}$ & Gaming \\
\hline $\begin{array}{l}\text { Sensors: senseBox } \\
\text { senseBox allows users and institutions to contribute their projects' sensor data. } \\
\text { The data are used to help answer scientific questions and support citizen science } \\
\text { projects from the local to the global. Data collected by senseBox can increase the } \\
\text { measurement capacity of various environmental factors and can facilitate } \\
\text { improved statistics in areas such as traffic, pollution, and climate }\end{array}$ & Sensing \\
\hline
\end{tabular}




\section{App Architecture}

There are many types of apps suited to different purposes, devices, and scientific fields. Most commonly, the term app is used for a mobile app, which can be installed on smartphones via the Apple (IOS) and Google (Android) app stores. However, in a broader sense, every type of software that can be installed on and run via a web browser is also an app. From a technical perspective, mobile apps and web apps are the most common in citizen science.

There are a wide range of mobile and web apps available - from mobile apps for monitoring and sharing observations on smartphones to image and video classification platforms to complex, interactive gaming apps.

There are also differences in how apps operate. Apps can be stand-alone software, managed by individual projects; or they can be based on an app platform that provides specific functionalities (not to be confused with citizen science platforms, the support infrastructure for citizen science projects; see Hai-Ying Liu et al., this volume, Chap. 22). Examples of app platforms include Zooniverse for image and video classification and the SPOTTERON platform which hosts map-based mobile apps. Furthermore, there are app platforms which operate just one app; these are often used for data collection - a practice generally employed in general species monitoring, one example being iNaturalist.

\section{Common Structure of a Citizen Science App Software Toolkit}

A common organisation scheme in interactive software is based on distinguishing between a front end (which users can see and interact with) and a back end (which only administrator accounts can access); see Fig. 23.1. This structure is also often used in citizen science toolkits and platforms. Generally speaking, the platform contains the core functionalities, but additional features can also be integrated. The system provides all the user functionality; users interact with it via a front end user interface. This front end can be accessed via a web browser (web app) or an app on a smartphone (mobile app).

Data contributions are stored in the platform's database. A database usually contains entries labelled by their ID, date, and category. An application programming interface (API) provides data access via defined parameters so data can be exchanged internally between the application server and the front end or externally with other servers. The API is also used to integrate external data in a citizen science toolkit, for example, sensors, which are prompted on a regular basis by the system.

Especially for more sensitive data, such as users' account information and personal data, safeguard mechanisms must be employed. Furthermore, all sensitive data traffic should be encrypted to protect the data from being accessed and misused.

While, for some citizen science apps, a constant Internet connection is required, others, such as field monitoring apps, also need to work offline. In that case, all 


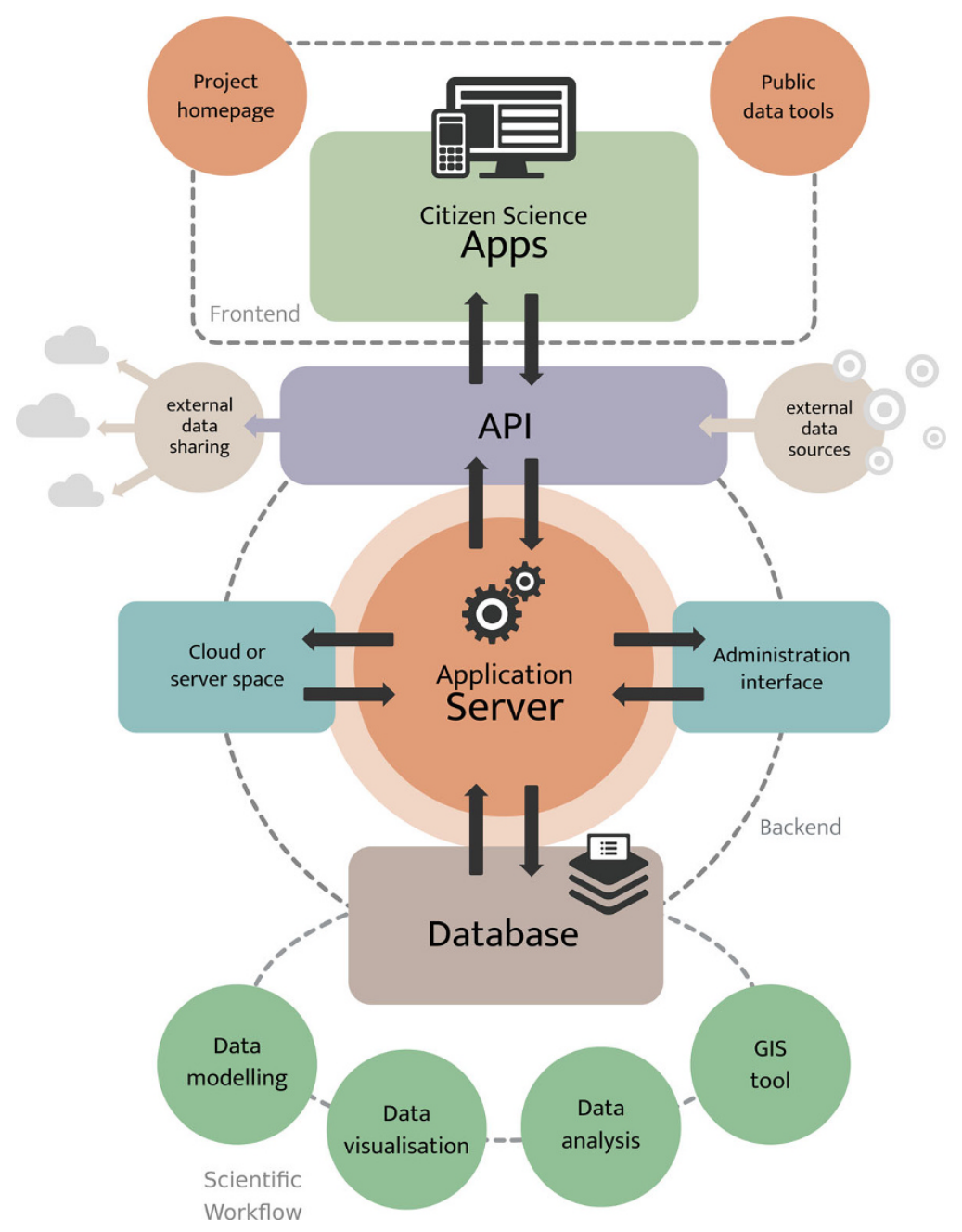

Fig. 23.1 An app/server system with a common online infrastructure

critical content and functionalities must be included in the app itself, and data contributions must be saved locally to be uploaded later. If an app is map based, an offline map download feature can sometimes also be required.

Due to the many different approaches, systems, and coding languages available to develop and run software, solutions can vary. This is underlined by the extensive inventory provided by Rieger and Majchrzak (2019) in which they compare mobile app tools, such as Cordova and PhoneGap, and languages such as AngularJS and HTML5/JS. Their comparison was based on the following criteria: infrastructure, development, hardware and software functionality, and usage. Despite the fact that it is hard to choose the best combination of tools for a specific application, the authors 
introduce weight profiles, which can be seen as common patterns of development and allow for a quick assessment of needs.

\section{How to Create and Maintain Citizen Science Apps}

The development of apps and digital tools can be a complex task. There are many aspects to consider, from technical development to design, from the server infrastructure to ongoing updates. The online technology environment changes rapidly, especially in the mobile sector. Rieger and Majchrzak (2019) refer to a 2-year period (2017-2019) in which mobile app development has shifted from a focus on smartphones and tablets to a wide range of devices, such as car-based technology and the Internet of Things (IoT). Personal data protection and security aspects must also be considered when planning to run citizen science apps.

Look and Feel Since citizen science is interactive by definition, the visual quality (look and feel) and the project identity (strong branding: a telling name with a quality logo, the wording, and the colours used) are essential elements when planning apps, websites, and dissemination activities (see Rüfenacht et al., this volume, Chap. 24). Since, in most citizen science projects, the aim is to include the public, having a strong project identity helps to communicate project objectives. While a high-quality look and feel can help to spark participant curiosity, app usability is also enhanced by definite forms of visual communication.

Re-use The best method of app development depends on the needs, goals, and target group/s of a project. If the required features are unique or highly specialised, the project will need to develop stand-alone apps. However, if the features required are relatively common in the field of citizen science, it is best not to reinvent the wheel. App platforms offer out-of-the-box functionalities and also provide app maintenance.

Co-creation Sometimes utilised by hackathons, co-creation can be used for prototyping, creating proof-of-concept versions of apps, and experimental approaches to developing specialised app functionalities. Hackathons are events where participants create software which can then be used more widely. Due to the limited time available when using a hackathon approach, it is vital to have a clear focus on functionality and also to plan for ongoing app updates. In the COST Action CA15212 Citizen Science to Promote Creativity, Scientific Literacy, and Innovation throughout Europe, ways have been explored to foster innovation in app development and learn from good practice. Therefore, it initiated a hackathon focused on citizen science app development (see Box 23.1). The main realisation that emerged from the preparation and execution of the hackathon is that while there are many apps relevant to citizen science, they are usually dedicated to a single purpose. Simultaneously, many apps have similar functionality, and there is a need for interoperability to let new developers build their apps by sharing software modules and data formats, so less time is spent reinventing the wheel. 


\section{Box 23.1: House of Apps: A COST Action Hackathon}

During June-August 2018, the COST Action CA15212 organised a hackathon aimed at the creation, findability, and re-use of mobile apps for citizen scientists (Lemmens and Antoniou 2019). App developers were asked to create small software components that focused either on interfacing mobile sensors or importing geodata (e.g. satellite images, in situ sensor data). Prior to the actual software development, project partners were asked to formulate suggested challenges (see Table 23.2 for examples).

The hackathon was concluded with a COST workshop at the OGRS symposium in Lugano, ${ }^{1}$ at which the winning app and the research undertaken were presented. This research led to the re-use of the app's components in the GEO-C Open City Toolkit (see also Pajarito and Gould 2018).

User Interface Design The design of a citizen science app is crucial for participation, motivation, and, also, data quality. Design connects function and form and defines how the various elements work together and how they are perceived when being used. Modern apps should have a clear and structured layout and wellinterchained functionalities that are presented in the user interface only when necessary. In general, the more on point an interface is, the more effective it becomes for frequent use. Apps can still have complex functionalities, but their interfaces must be user-friendly. This principle is also essential for the input dialog used to add data to a citizen science project. The input dialog is established by app menus and user input (text, pictures, voice, etc.) and determines what a participant can and cannot contribute via the app. A hierarchical structure with visual representations for options and manageable selection lists facilitates straightforward participation and reduces data input errors.

Participant Motivation It is important to understand the target users and their needs and desires. These have a significant influence on participant motivation. For example, depending on the participants and task community, app features can help maintain long-term motivation. In order to achieve this, usability must also be maintained. Thus, apps need a plan to ensure constant maintenance and updates. This includes security updates and constant privacy protection. Equally important is the scientific perspective. Data and API standards can provide valuable guidelines on how data should be stored and made accessible.

Supporting Infrastructure The technical infrastructure needed to run citizen science apps must also be considered. Due to their interactivity, data security and performance of the servers are both essential infrastructure components. The protection of users' personal data is also affected: everything, from data hosting to map servers, should run on professionally hosted infrastructure (see also Hai-Ying Liu

\footnotetext{
${ }^{1}$ http://2018.ogrs-community.org/
} 
Table 23.2 A selection of mobile app challenges for the COST hackathon. The app functionality needs set out the requirements for developing the app

\begin{tabular}{|c|c|c|}
\hline Theme - Title & Objective & App functionality needs \\
\hline $\begin{array}{l}\text { The networking } \\
\text { citizen - 'How } \\
\text { unusual is it?' }\end{array}$ & $\begin{array}{l}\text { An app that explores whether an obser- } \\
\text { vation is common or unusual (out of the } \\
\text { normal range of measurement). Pro- } \\
\text { vides immediate feedback based on } \\
\text { frequency of occurrence in social net- } \\
\text { works; this should reinforce } \\
\text { participation }\end{array}$ & $\begin{array}{l}\text { Intensity of the phenomenon } \\
\text { (e.g. fish size, number of fish, } \\
\text { rainfall measurements) } \\
\text { Time stamp (date) } \\
\text { Location } \\
\text { Location attributes (e.g. land use) }\end{array}$ \\
\hline $\begin{array}{l}\text { Health - 'Promot- } \\
\text { ing sustainable } \\
\text { traffic' }\end{array}$ & $\begin{array}{l}\text { An app that collects data about pedes- } \\
\text { trian and cyclist usage of roads, pave- } \\
\text { ments, and trails, with an emphasis on } \\
\text { understanding which elements disturb } \\
\text { the flow of traffic. The aim of this app is } \\
\text { to provide data for planners to help } \\
\text { design and manage a sustainable trans- } \\
\text { portation network for pedestrians and } \\
\text { cyclists }\end{array}$ & $\begin{array}{l}\text { Route used (GPS location, ele- } \\
\text { vation) and time } \\
\text { Number of stops } \\
\text { Number of accidents for bikes } \\
\text { and pedestrians (if known) } \\
\text { Subjective feeling of security and } \\
\text { route efficiency } \\
\text { Active reporting of obstacles and } \\
\text { hazards } \\
\text { Measuring of light conditions } \\
\text { Parking places } \\
\text { Time of year and weather } \\
\text { Uploading photos of obstacles, } \\
\text { places, and vistas } \\
\text { Measuring noise levels }\end{array}$ \\
\hline $\begin{array}{l}\text { Land use - Land } \\
\text { use camera } \\
\text { function }\end{array}$ & $\begin{array}{l}\text { A software library that will handle } \\
\text { smartphone cameras for land use and } \\
\text { land cover apps }\end{array}$ & $\begin{array}{l}\text { GPS awareness } \\
\text { Show the line of the horizon } \\
\text { accurately } \\
\text { Zoom level awareness } \\
\text { Calculate sky percentage } \\
\text { Flash awareness } \\
\text { Provide information and tips to } \\
\text { the user before photo capturing } \\
\text { Panorama mode } \\
\text { Resolution awareness }\end{array}$ \\
\hline
\end{tabular}

https://cs-eu.net/news/house-apps-create-great-apps-citizens

et al., this volume, Chap. 22). If free services offered by commercial corporations are put to use in citizen science projects, significant conflict can arise. Free services such as online forms, maps, and even analytics tools are not paid for with money, but with users' personal data that is used for profiling and targeted advertising.

Testing After the app design and development processes, it is important to plan for extensive testing and bug fixing that can be in place when an app launches. Regular app updates are also essential. Mobile software development deals with a fastchanging environment, and software, like apps, must be able to adapt to that environment on an ongoing basis. Planning for ongoing maintenance is especially important when creating stand-alone apps. On citizen science app platforms, the system is usually already established and been proven to work successfully in other public citizen science apps. 
Maintenance The life cycles of online software and, especially, mobile apps, are short, usually measured in weeks rather than months. To prevent citizen science apps from becoming outdated and unusable or losing functionality, ongoing development and regular updates are required. App updates can deliver bug fixes and improvements; they are essential for ongoing security, stability, and compatibility over a project's runtime. For stand-alone apps, the runtime costs can easily exceed the development costs; on citizen science app platforms, app updates are usually provided automatically.

\section{Future of Apps in Citizen Science}

In parallel with the use of current technologies, citizen science project stakeholders should maintain a forward-looking mindset regarding technological advances. With technology it is not uncommon to experience changes and disruptions in existing processes, which can considerably alter the way they are dealt with (such as how data is gathered or analysed). This, in combination with the fact that hardware is progressively improving and becoming more affordable, alongside advances in open-source software, points to a promising future for the use of new technologies in citizen science.

In this context, citizen science projects can aim to achieve two important goals. First, they should take advantage of existing technologies that are used in other domains. Migrating effective technologies and applications from other domains can enable citizen science projects to be more productive and simplify participant activities. For example, adoption of existing technologies can improve several aspects of citizen science projects, such as the ability to increase data collection through newly available sensors (Plageras et al. 2018); enhance user engagement and participation through gamification (Antoniou and Schlieder 2014); improve and manage data quality through machine learning (ML) and artificial intelligence (AI) algorithms (Zhang et al. 2018); and create more collaborative environments by using social networking approaches (Liao et al. 2015).

Adoption of existing technologies paves the way to achieve the second goal: enabling citizen science projects to be able to absorb new technological advances and developments and, thus, to ensure that citizen science will not be left behind in the technological race. There is real danger of creating a technological gap in future citizen science projects - which is tempting to excuse or disguise behind the 'citizen' element of a citizen science project. In other words, citizen science projects need to stay at the forefront of technological advances in order to be in a position to adopt future developments. If citizen science projects try to develop by relying only on volunteer strength, they will position themselves in a situation where emerging technologies and applications will be difficult, if not impossible, to adopt. Furthermore, citizen science project research should be on a par with research that take place purely in the academic arena. To achieve this parity, citizen science projects should aim for technological proficiency and excellence. Technologies and apps related to 
cloud computing, IoT, and AI need to become mainstream for citizen science projects, rather than being the reserve of flagship projects.

In this context, and despite the obvious challenge of predicting the future of apps in citizen science, the discussion will turn to what are deemed the most influential technological factors in citizen science: AI and ML (see also Franzen et al., this volume, Chap. 10). The hypothesis that AI and ML will lead future developments in citizen science is based on recent breakthroughs that have been achieved in several domains. Examples can be found in earth observation and remote sensing tasks (Ma et al. 2019) and in quality assurance ( $\mathrm{Li}$ et al. 2017). In general, when the availability of huge volumes of data can threaten to overwhelm human efforts to meaningfully analyse or understand useful patterns and rules, AI and ML can provide considerable advantages.

Indeed, globally, citizen science projects are gathering large volumes of citizencontributed data and observations (e.g. iNaturalist, which at the time of writing, has achieved almost 30 million observations in almost 250 thousand categories, contributed by more than a million citizens). Tasks such as observation categorisation (and, as a result, data quality) are primarily based on the unverified contributions of participants (especially of newcomers) or verified by groups of moderators that act as quality filters. While this approach has been successful in a number of citizen science projects, the addition of AI and ML can help significantly by 'learning' to recognise species to assign them to the correct category or to suggest the most plausible choice to participants.

This generic example can be applied with varying degrees to several other citizen science projects or tasks. These include the correction of raster data through noise removal (Wolterink et al. 2017), the completion of missing data (Turabieh et al. 2018), and spotting outliers and anomalies in data sets (Zhou and Paffenroth 2017).

The introduction of AI and ML in citizen science projects is not meant to replace humans. For various reasons (Gilmer et al. 2018), including the immaturity of the AI and ML fields (Heaven 2019), humans are still needed in the decision-making process to provide their intuition, imagination, and reasoning, which are not possible to mimic via $\mathrm{AI}$ or ML algorithms. However, as $\mathrm{AI}$ and $\mathrm{ML}$ are becoming increasingly accessible, the collaboration of AI and ML can provide benefits in time, resources, and effort needed for both simple and critical tasks (Antoniou and Potsiou 2020). Moreover, the intertwining of volunteerism with the power of AI and ML can boost citizen science projects' effectiveness in user engagement and project usability. User engagement can be enhanced and increased through AI and ML processes, which can facilitate certain tasks and make apps more responsive and contributions less error-prone. Project usability can be enhanced by improving overall data quality that, in turn, will encourage the use of citizen science data sets by multiple stakeholders while at the same time free manpower for other tasks that need human input.

The next big step in the development of the future citizen science apps will likely be the mainstream incorporation of AI and ML-trained models in mobile citizen science apps that will be able to infer or suggest options to participants with high accuracy levels. In such an AI and ML-enabled future, the possibilities for citizen science projects are countless and the combination of human effort and mobile apps will become the centre of gravity for every project. 
Mobile apps rapidly adopt technology developments. Typically, maintenance cycles measured in weeks are not uncommon. Especially in citizen science, mobile apps play an important role as the extended sensor of the user. Practice has shown that key aspects should be considered for the successful development of apps in citizen science, such as usability, user interaction, and interoperability. Just as in any other aspect of a citizen science project, the development of apps should also focus on participants' requirements. The performance, usability, a high-quality look and feel, and interface design are main keys for success. As computing power is rapidly increasing in mobile devices, so are the possibilities to use high-end software functionality, implementing AI and ML-driven features. This may even change the role of citizens in citizen science, as certain tasks will be done automatically.

\section{References}

Ambrose-Oji, B., van der Jagt, A. P. N., \& O’Neil, S. (2014). Citizen science: Social media as a supporting tool. Edinburgh: Forest Research.

Antoniou, V., \& Potsiou, C. (2020). A deep learning method to accelerate the disaster response process. Remote Sensing, 12(3), 544.

Antoniou, V., \& Schlieder, C. (2014). Participation patterns, VGI and gamification. Presented at the proceedings of AGILE 2014.

Bonney, R., Shirk, J. L., Phillips, T. B., Wiggins, A., Ballard, H. L., Miller-Rushing, A. J., \& Parrish, J. K. (2014). Citizen science. Next steps for citizen science. Science, 343(6178), 1436-1437. https://doi.org/10.1126/science.1251554.

Bowser, A., Hansen, D., He, Y., Boston, C., Reid, M., Gunnell, L., \& Preece, J. (2013). Using gamification to inspire new citizen science volunteers. In Gamification '13: Proceedings of the first international conference on gameful design, research, and applications. https://doi.org/10. $1145 / 2583008.2583011$.

ECSA. (2015). Ten principles of citizen science. Available at: https://ecsa.citizen-science.net/sites/ default/files/ecsa_ten_principles_of_citizen_science.pdf

Gilmer, J., Adams, R. P., Goodfellow, I., Andersen, D., \& Dahl, G. E. (2018). Motivating the rules of the game for adversarial example research. arXiv preprint arXiv:1807.06732. https://arxiv. org/pdf/1807.06732.pdf

Haklay, M. (2013). Citizen science and volunteered geographic information: Overview and typology of participation. In D. Sui, S. Elwood, \& M. Goodchild (Eds.), Crowdsourcing geographic knowledge (pp. 105-122). Dordrecht: Springer.

Heaven, D. (2019). Why deep-learning AIs are so easy to fool. Nature, 574(7777), 163.

Lemmens, R., \& Antoniou, V. (Eds.) (2019). Hackathon report: House of Apps: Create great apps for citizens. Citizen Science COST Action CA15212.

Li, B. H., Hou, B. C., Yu, W. T., Lu, X. B., \& Yang, C. W. (2017). Applications of artificial intelligence in intelligent manufacturing: A review. Frontiers of Information Technology \& Electronic Engineering, 18(1), 86-96.

Liao, Y. W., Huang, Y. M., Chen, H. C., \& Huang, S. H. (2015). Exploring the antecedents of collaborative learning performance over social networking sites in a ubiquitous learning context. Computers in Human Behavior, 43, 313-323.

Luna, S., Gold, M., Albert, A., Ceccaroni, L., Claramunt, B., Danylo, O., et al. (2018). Developing mobile applications for environmental and biodiversity citizen science: Considerations and recommendations. In A. Joly, S. Vrochidis, K. Karatzas, A. Karppinen, \& P. Bonnet (Eds.), Multimedia tools and applications for environmental \& biodiversity informatics (pp. 9-30). Cham: Springer. 
Ma, L., Liu, Y., Zhang, X., Ye, Y., Yin, G., \& Johnson, B. A. (2019). Deep learning in remote sensing applications: A meta-analysis and review. ISPRS Journal of Photogrammetry and Remote Sensing, 152, 166-177.

Newman, G., Wiggins, A., Crall, A., Graham, E., Newman, S., \& Crowston, K. (2012). The future of citizen science: Emerging technologies and shifting paradigms. Frontiers in Ecology and the Environment, 10(6), 298-304. https://doi.org/10.1890/110294.

Pajarito, D., \& Gould, M. (2018). Mapping frictions inhibiting bicycle commuting. ISPRS International Journal of Geo-Information, 7(10), 396. https://doi.org/10.3390/ijgi7100396.

Pejovic, P., \& Skarlatidou, A. (2020). Understanding interaction design challenges in mobile extreme citizen science. International Journal of Human-Computer Interaction, 36(3), 251-270. https://doi.org/10.1080/10447318.2019.1630934.

Plageras, A. P., Psannis, K. E., Stergiou, C., Wang, H., \& Gupta, B. B. (2018). Efficient IoT-based sensor BIG Data collection - Processing and analysis in smart buildings. Future Generation Computer Systems, 82, 349-357.

Rieger, C., \& Majchrzak, T. (2019). Towards the definitive evaluation framework for crossplatform app development approaches. Journal of Systems and Software, 153, 175-199. https://doi.org/10.1016/j.jss.2019.04.001.

Silvertown, J. (2009). A new dawn for citizen science. Trends in Ecology and Evolution, 24(9), 467-471. https://doi.org/10.1016/j.tree.2009.03.017.

Sturm, U., Luna, S., Albert, A., Schade, S., \& Kasperowski, D. (Eds.) (2017). Defining principles and guidelines for mobile apps and platform development for best practice in citizen science. Berlin, December 13-14, 2016. Report of the workshop. https://ecsa.citizen-science.net/sites/ default/files/report_of_the_workshop.pdf

Teacher, A. G. F., Griffiths, D. J., Hodgson, D. J., \& Inger, R. (2013). Smartphones in ecology and evolution: A guide for the app-rehensive. Ecology and Evolution, 3(16), 5268-5278. https://doi. org/10.1002/ece3.888.

Trojan, J., Schade, S., Lemmens, R., \& Frantál, B. (2019). Citizen science as a new approach in geography and beyond: Review and reflections. Moravian Geographical Reports, 27(4), 254-264. https://doi.org/10.2478/mgr-2019-0020.

Turabieh, H., Salem, A. A., \& Abu-El-Rub, N. (2018). Dynamic L-RNN recovery of missing data in IoMT applications. Future Generation Computer Systems, 89, 575-583.

Wolterink, J. M., Leiner, T., Viergever, M. A., \& Išgum, I. (2017). Generative adversarial networks for noise reduction in low-dose CT. IEEE Transactions on Medical Imaging, 36(12), 2536-2545.

Wynn, J. (2017). Citizen science in the digital age. Rhetoric, science and public engagement. Tuscaloosa: The University of Alabama Press.

Zhang, Q., Yang, L. T., Chen, Z., \& Li, P. (2018). A survey on deep learning for big data. Information Fusion, 42, 146-157.

Zhou, C., \& Paffenroth, R. C. (2017). Anomaly detection with robust deep autoencoders. In Proceedings of the 23rd ACM SIGKDD international conference on knowledge discovery and data mining (pp. 665-674). New York: ACM.

Rob Lemmens works in the Faculty of Geo-Information Science and Earth Observation (ITC) at the University of Twente, with a research focus on semantic web technologies and the improvement of geo-crowdsourcing methods.

Vyron Antoniou is an officer in the Hellenic Army Geographic Directorate. His background is in GIS, VGI, web mapping applications, survey engineering, cartography, and terrain analysis. His current interests are centred on machine learning and deep learning for geospatial applications. 
Philipp Hummer has been fascinated by nature since his childhood when he had his first citizen science experience, reporting European green toad observations for herpetology research. In 2015, after a 15-year career in media design, Philipp founded ?SPOTTERON?, a platform for citizen science apps and toolkits with a focus on design, interactivity, and community engagement.

Chryssy Potsiou is a professor in the School of Rural \& Surveying Engineering, NTUA, in the fields of cadastre, property valuation, and spatial information management. She is honorary president of the International Federation of Surveyors and vice chair of WPLA. She has also served as member of the Greek Cadastral Agency board and a member of the UN ECE Working Party for Land Administration.

Open Access This chapter is licensed under the terms of the Creative Commons Attribution 4.0 International License (http://creativecommons.org/licenses/by/4.0/), which permits use, sharing, adaptation, distribution and reproduction in any medium or format, as long as you give appropriate credit to the original author(s) and the source, provide a link to the Creative Commons license and indicate if changes were made.

The images or other third party material in this chapter are included in the chapter's Creative Commons license, unless indicated otherwise in a credit line to the material. If material is not included in the chapter's Creative Commons license and your intended use is not permitted by statutory regulation or exceeds the permitted use, you will need to obtain permission directly from the copyright holder.

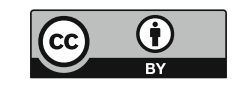

